\title{
Environmental NMR: Fast-field-cycling Relaxometry
}

\author{
Pellegrino Conte \& Giuseppe Alonzo \\ Università degli studi di Palermo, Palermo, Italy
}

Fast-field-cycling (FFC) NMR relaxometry deals with the variation of the spin-lattice relaxation times $\left(T_{1}\right)$ in a complex system, as the strength of the applied magnetic field is changed. Information about molecular dynamics can be achieved. Until now, only model theories for FFC NMR relaxometry have been developed for polymer and material sciences. Just a few applications have been performed in the environmental sciences. These mainly deal with soil porosity, rock permeability, biomass transformations, and natural organic matter dynamics. Further, FFC NMR relaxometry can also be applied to monitor the environmental fate of contaminants, to understand the dynamics of nutrients at the soil-plant interface, and to evaluate reaction mechanisms in heterogeneous catalysis for the development of green reactions. This article summarizes the advances of the technique in environmental investigations and describes the tools used to monitor dynamics of organic and inorganic molecules in environmental compartments.

Keywords: fast-field cycling, NMR, relaxometry, environmental field cycling, spin-lattice relaxation

How to cite this article:

eMagRes, 2013, Vol 2: 389-398. DOI 10.1002/9780470034590.emrstm1330

\section{Introduction}

NMR relaxometry refers to the ensemble of techniques applied to monitor how fast nuclear spin magnetization switches from a nonequilibrium state to the equilibrium distribution. This phenomenon, referred to as relaxation, arises from fluctuating local magnetic or electrical fields that are generated by nuclear dipoles, unpaired electrons, electric charges interacting with nuclear quadrupole moments for $>1 / 2$ spin nuclei, anisotropy of the chemical shielding tensor, fluctuating scalar coupling interactions, and molecular rotations. Mostly, fluctuations are the result of molecular motions. For this reason, measurement of the relaxation times (or relaxation rates that are the inverse of the relaxation times) can be related to molecular dynamics. ${ }^{1-4}$ The frequency range of the microscopic molecular fluctuations is comprised within the interval of $10-10^{9} \mathrm{~Hz} \cdot{ }^{5}$ Because of this, relaxation time values range from few picoseconds to seconds.

Two different relaxation times are associated to nuclear relaxation. The first one, indicated as longitudinal or spin-lattice relaxation time $\left(T_{1}\right)$, measures the time needed for the recovery of the longitudinal component of the magnetization along the $z$-axis. The second relaxation time, known as transversal or spin-spin relaxation time $\left(T_{2}\right)$, measures the decaying for the transversal component of the magnetization to zero. In particular, frequency motions from $<10$ to $\approx 10^{4} \mathrm{~Hz}$ are monitored by diffusimetry, transverse relaxometry, and residual spin coupling NMR techniques; motions with frequency ranging between $\approx 10^{4}$ and $\approx 10^{5} \mathrm{~Hz}$ are investigated by rotating frame NMR relaxometry experiments; motion frequencies from $\approx 10^{5}$ to $\approx 10^{8} \mathrm{~Hz}$ are observed by field-cycling NMR relaxometry; and frequencies of $>10^{8} \mathrm{~Hz}$ are monitored using traditional high field NMR spectrometers. ${ }^{5,6}$
Field-cycling NMR investigates molecular motions through the measurement of the variations of the longitudinal relaxation times, as the external magnetic field intensity $\left(B_{0}\right)$ is changed. Field cycling can be achieved by the 'sample shuttle technique' (SST). This consists in moving pneumatically the sample through different positions in a magnetic field, where different flux densities can be recognized. ${ }^{6}$ Typical SST field switching times (SWTs) are of the order of hundreds of milliseconds. This limits the application of the technique to samples with longitudinal relaxation time values $>100 \mathrm{~ms}$. A better solution for measuring samples with $T_{1}$ values $<100 \mathrm{~ms}$ is the application of fast-field cycling (FFC). In this case, field switching is achieved electronically by modulating the current passing through the coil generating the magnetic field. Owing to the development of modern electronics, field switching can be automatically achieved in $2-3 \mathrm{~ms}$ (that is around $30-50$ times lower than in SST method), thereby allowing to monitor longitudinal relaxation times of the order of $2 \mathrm{~ms} .{ }^{6,7}$ The high-frequency limits for FFC NMR relaxometry depend on the available high field magnets. Conversely, the low-frequency limits are governed by small fluctuations of magnetic field because of, for example, the Earth's field or any other magnetic stray field, and $T_{1}$ values that are of the order of the SWT. ${ }^{6,8}$ Relaxation times shorter than the SWTs can also be measured if special electronic precautions are applied. ${ }^{9}$

This article highlights FFC NMR relaxometry. The nature of the FFC NMR relaxometry experiment will be described and recent advances in environmental applications will be provided. It must be stated that the technique can be applied, in principle, on all the NMR visible nuclei. ${ }^{10}$ However, until now, to the best of our knowledge, all published studies focus only on ${ }^{1} \mathrm{H}$ when FFC NMR relaxometry is applied in environmental 
investigations. For this reason, from now on, we refer only to FFC NMR behavior of protons. Finally, the readers are referred to wider and more specific review papers to deep knowledge on the theory and the instrumental details behind the technique. ${ }^{5,6,8,11}$

\section{The FFC NMR Relaxometry Experiment in Practice}

Figure 1 shows the typical FFC NMR experimental design based on a pre-polarized (PP) and a nonpolarized (NP) sequences. ${ }^{6}$ Namely, three steps can be recognized: polarization, relaxation, and acquisition. During the first step of the PP sequence (Figure 1a), the longitudinal magnetization is generated through the application of a polarization field $\left(B_{\mathrm{POL}}\right)$ for a limited and fixed period of time (indicated as polarization time, $\left.T_{\mathrm{POL}}\right)$. Afterwards, the magnetic field is switched to a

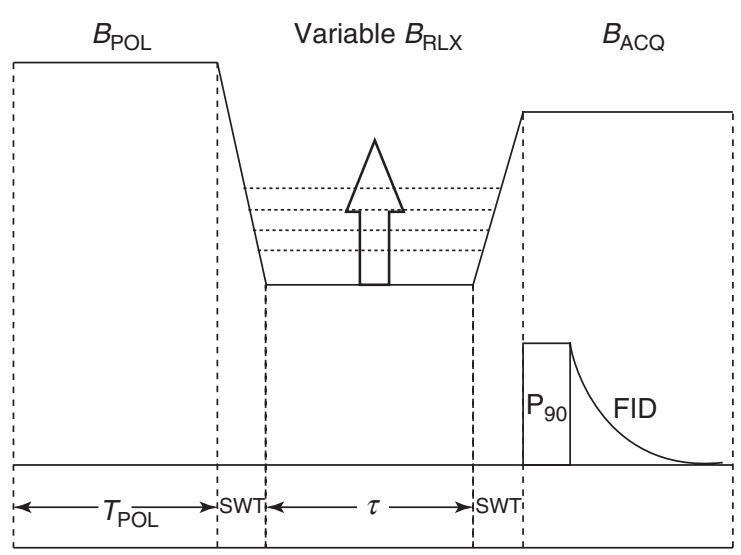

(a) new one (the relaxation field, $B_{\mathrm{RLX}}$ ), applied for a period $\tau$ during which the magnetization intensity relaxes to reach a new equilibrium condition. Finally, the application of a ${ }^{1} \mathrm{H}$ $90^{\circ}$ pulse into an acquisition magnetic field $\left(B_{\mathrm{ACQ}}\right)$ held for a fixed time, makes the magnetization observable and the free induction decay (FID) acquirable. In the NP sequence, $B_{\mathrm{POL}}$ is null (Figure 1b). The PP sequence is applied when the relaxation field becomes very low in intensity and enhancement of sensitivity is needed for FID achievement. ${ }^{11}$ The crossover field between NP and PP sequences is approximately retrieved when the relaxation field intensity is half of that of the polarization field. ${ }^{11}$

The FIDs obtained by arraying the $\tau$ values applied during the relaxation step provide the block scheme shown in Figure 2(a) and (b) for the NP and the PP sequences, respectively. Each block represents the FID at any of the arrayed $\tau$ values. After measurement of FID areas, the block schemes are transformed

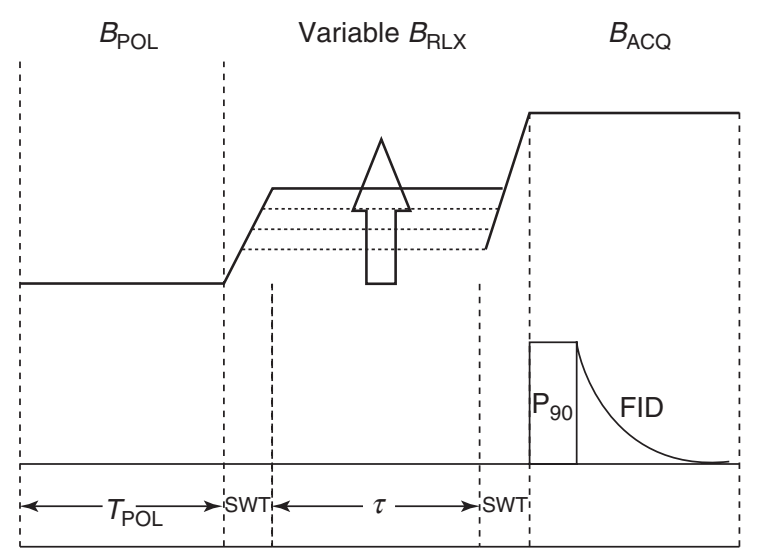

(b)

Figure 1. Pre-polarized (a) and nonpolarized (b) sequences used in fast field cycling NMR relaxometry experiments. $B_{\mathrm{POL}}$ is the polarization field applied for the period $T_{\mathrm{POL}}, B_{\mathrm{RLX}}$ is the relaxation field applied for the variable $\tau$ time, and $B_{\mathrm{ACQ}}$ is the acquisition field. FID is the free induction decay, $\mathrm{P}_{90}$ is the $90^{\circ}$ pulse, and SWT is the switching time needed to change the intensity of the magnetic field. $B_{\mathrm{POL}}$ intensity is null in the NP sequence

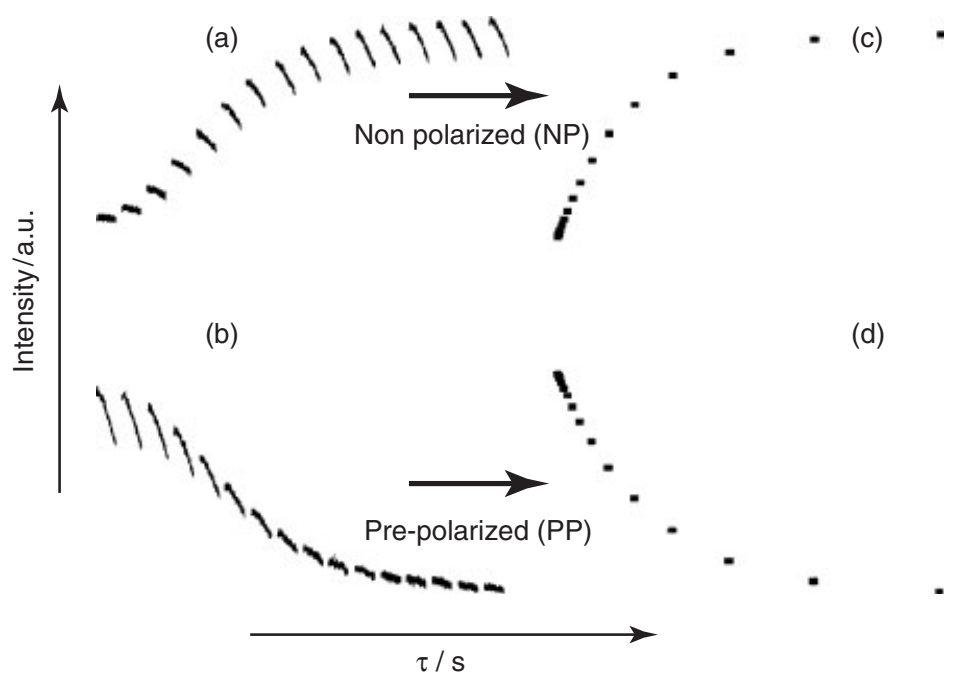

Figure 2. Multiblock FID sequences obtained by applying (a) the NP and (b) the PP schemes. The integration of the FID in each block provides (c) the recovery and (d) the decay curves, whose fitting allows achievement of $T_{1}$ values 
in a recovery (Figure 2c) or decay (Figure 2d) curve according to the nature of the sequence applied (NP and PP, respectively).

\section{Elaboration of the Decay/Recovery Curves for Natural Systems}

Both the recovery and decay curves (Figure 2) describe the evolution of the ${ }^{1} \mathrm{H}$ longitudinal magnetization $[M(\tau)]$ in the relaxation field when this is held for an arrayed time $\tau$. The magnetization in the relaxation field is aligned along the $B_{\mathrm{RLX}}$ direction and its value equals that in the polarization field when $\tau=0^{5}$ :

$$
M(0)=M_{z}(0)=M_{0}\left(B_{\mathrm{POL}}\right)
$$

Equation (1) is valid only when the evolution of the magnetization is negligible during the SWT. When $\tau \neq 0$, the magnetization evolves according to equation $(2)^{5}$ :

$$
\begin{aligned}
M_{z}(\tau)= & M_{0}\left(B_{\mathrm{RLX}}\right)+\left[M_{0}\left(B_{\mathrm{POL}}\right)-M_{0}\left(B_{\mathrm{RLX}}\right)\right] \\
& \times \exp \left[\frac{-t}{T_{1}\left(B_{\mathrm{RLX}}\right)}\right]
\end{aligned}
$$

At the end of the relaxation period, the magnetization is detected by applying a third magnetic field and a $90^{\circ}$ pulse, as outlined earlier. According to equation (2), the evolution of the magnetization is a recovery curve in the NP condition (Figure 2c), whereas it is a decay curve in the PP condition (Figure 2d).

In equation (2), $T_{1}$ is the spin-lattice relaxation time, which depends on $B_{\mathrm{RLX}}$ intensity. $T_{1}$ represents the lifetime of the first-order rate process that returns the spin magnetization to the Boltzmann equilibrium along the $z$-axis. ${ }^{12}$ Its value depends on both the molecular dynamics of the investigated system $^{12}$ and the frequency of the applied magnetic field. ${ }^{6}$ In fact, the equilibrium recovery of the longitudinal magnetization (and, hence, the time, $T_{1}$, needed for that) is determined by the presence in the lattice of magnetic fields oscillating at a frequency corresponding to that of the transition between the nuclear energetic levels. All the nuclear Larmor frequencies are affected by the applied magnetic field. For this reason, only the molecular motions oscillating at the frequency of the specific magnetic field value are effective in promoting nuclear relaxation. The same motions could not be effective for a different value of the applied magnetic field. Molecular motions strongly affect dipolar interactions. In particular, the faster the motions are, the lower is the dipolar interaction efficiency, thereby favoring longer $T_{1}$ values. Conversely, slower molecular dynamics can be associated with shorter spin-lattice relaxation times because of stronger nuclear dipolar interactions. ${ }^{12}$

According to Canet, ${ }^{13}$ the relaxation decay/recovery curves at each relaxation field intensity are described by the sum of exponential functions as indicated in equation (3):

$$
M(\tau)=a+\sum_{i=1}^{N}\left[b_{i} \times \exp \left(\frac{-\tau}{T_{1 i}}\right)\right]
$$

Here, $a$ is the offset and $b_{i}$ is the magnetization intensity at the Boltzmann equilibrium of the $i$ th component of the molecular motion at each fixed $B_{\mathrm{RLX}}$ intensity.

Equation (3) is monoexponential only when homogeneous molecular fluctuations are present in simple systems such as pure solvents. ${ }^{14}$ Conversely, a multiexponential behavior must be accounted for when complex mixtures are investigated. ${ }^{13,15-25}$ As an example, Figure 3 reports a decay curve obtained at $0.25 \mathrm{mT}$ for a water-saturated poplar char whose dynamics has been described by De Pasquale et al. ${ }^{22}$ and Conte et al. ${ }^{23}$ The monoexponential form of equation (3) (dashed line in Figure 3) failed in fitting the data for $\tau$ values $>0.15 \mathrm{~ms}$, whereas the biexponential form (continuous line in Figure 3) was successful within the whole range of $\tau$ values applied during the analysis. The presence of two different $T_{1}$ components indicates that the motion of the water molecules on the surface of the porous solid phase is more restricted

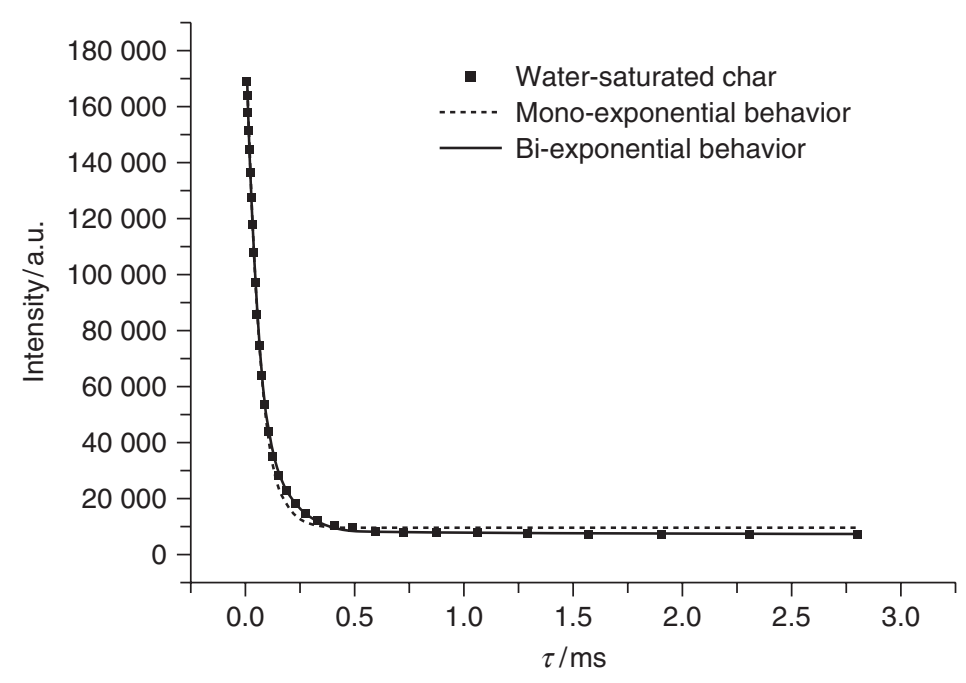

Figure 3. Example of a decay curve obtained at $0.25 \mathrm{mT}$ for a water-saturated biochar. The dashed and continuous lines are the fitting retrieved by applying the mono- and biexponential forms of equation (3), respectively 
than that of the bulk water (see also the section titled 'From $\tau$ Domain to $T_{1}$ Domain: the Inverse Laplace Transformation and the Relaxograms' for further details).

The limiting factor regarding equation (3) is the necessity to make assumptions on the number of exponential components to be used for the fitting of the experimental data. In fact, the larger the number of components is, the better is the quality of the fitting (e.g., higher $R^{2}$ and lower $\chi^{2}$ values). However, relaxometry measurements are also affected by an experimental noise that, in turn, influences the fitting error on the estimated $T_{1}$ values. In particular, the higher is the number of exponential components in equation (3), the larger is the fitting $T_{1}$ error, thereby making difficult the evaluation of the molecular dynamics of complex mixtures.

The stretched function given in equation (4) has been used instead of equation (3) in some papers dealing with environmental samples. ${ }^{20,22-26}$ Here, $a$ and $b$ have the same meaning as in equation (3), and $k$ is a heterogeneity parameter related to the stretching of the decay/recovery process:

$$
M(\tau)=a+b \times \exp \left[-\left(\frac{\tau}{T_{1}}\right)^{k}\right]
$$

This function, which accounts for the large sample heterogeneity resulting in a multiexponential behavior of the decay/recovery curves, ${ }^{27}$ can be considered as a superposition of exponential contributions, thereby describing the likely physical picture of some distribution in $T_{1}$. Equation (4) has the advantage that it is able to handle a wide range of behaviors within a single model. For this reason, assumptions about the number of exponentials to be used in modeling relaxometry data are not necessary.

\section{From $\tau$ Domain to $I_{1}$ Domain: The Inverse Laplace Transformation and the Relaxograms}

The recovery/decay curves (Figure $2 \mathrm{c}$ and $\mathrm{d}$ ) and the related distributions of exponential components [equations (3) and (4)] vary greatly in character and complexity in multiphase systems such as soils, ${ }^{17,19,24}$ sediments, ${ }^{25}$ plant tissues, ${ }^{15,18,20}$ natural organic matter, ${ }^{16,28}$ char, ${ }^{22,23}$ and new pesticide formulations. ${ }^{21}$ When different components of the molecular dynamics in these multiphase frames are described by longitudinal relaxation times with values very close to each other, a better representation of their distribution is attained by applying an inverse Laplace transformation in the form of equation $(5)^{29-33}$ :

$$
M(\tau)=\int_{T_{1 \min }}^{T_{1 \max }} D\left(T_{1}\right) \times \exp \left[-\left(\frac{\tau}{T_{1}}\right)\right] d\left(T_{1}\right)+\sigma
$$

Here, $T_{1 \min }$ and $T_{1 \max }$ are the slowest and the longest spin-lattice relaxation times within which all the $T_{1}$ values fall; $D\left(T_{1}\right)$ is the distribution function, which must be determined by solving equation (5); and $\sigma$ is an unknown noise component. The latter term renders impossible to find the exact distribution of relaxation times, thereby allowing infinite possible $D\left(T_{1}\right)$ solutions for equation (5). In order to find the most probable $D\left(T_{1}\right)$ distribution, some constraints must be used such as smoothness of the solution and variance of the experimental data. The algorithms developed to account for equation (5) are CONTIN ${ }^{29,30}$ (CONTINuous distribution) and UPEN ${ }^{31-33}$ (Uniform PENalty regularization). They differ between each other for the smoothing procedure used during the application. A detailed description of the two algorithms is out of the aims of the present article. The readers are addressed to specific reports ${ }^{29-33}$ that provide more details for the different approaches.

Regardless of the procedure used to reveal the most probable distribution of relaxation times, either CONTIN or UPEN algorithms provide similar $T_{1}$ distributions also referred to as relaxograms. Figure 4 shows, as an example, the relaxograms retrieved by applying the UPEN algorithm on the recovery curves acquired at a relaxation field of $200 \mathrm{mT}$ on conifer and poplar water-saturated chars. ${ }^{22}$ Both relaxograms are broad and complex, spanning several $T_{1}$ decades with the width of the signal $(A)$ decreasing in the order $A_{\text {(poplar char) }}>A_{\text {(conifer char) }}$. Spin-lattice relaxation time values of water on porous media surfaces are related to porosity. In fact, the motion of water confined in small-sized porous is more restricted than that moving in larger porous. For this reason, inter- and intramolecular interactions are stronger in smaller sized pores than in larger ones, thereby leading to the conclusion that pore-size distribution in the poplar char is wider than in conifer char. ${ }^{22}$

Relationships between pore sizes and $T_{1}$ distributions have been used to distinguish among different natural soils, ${ }^{19,24,34}$ to evaluate properties of natural and synthetic hydrated sands, ${ }^{17}$ to reconstruct the environmental evolution of saltmarsh sediments, ${ }^{25}$ to identify differences in plant tissues involved in soil development, ${ }^{20}$ to investigate the interactions between natural organic matter and clay minerals, ${ }^{28}$ and to monitor interactions between soil organic matter and cation-treated soils. ${ }^{35}$ Moreover, ${ }^{1} \mathrm{H} T_{1}$ relaxograms were also successfully applied to follow cellulose degradation in phosphoric acid with the aim to suggest new possible solvents for biomass transformations to biofuels. ${ }^{36}$

\section{The Nuclear Magnetic Resonance Dispersion Profiles: Quantitative Aspects in Environmental Applications}

Nuclear magnetic resonance dispersion (NMRD) curves are obtained by reporting longitudinal relaxation rates $\left(R_{1}=1 / T_{1}\right)$ versus the $B_{\mathrm{RLX}}$ intensities applied during the FFC NMR experiment. NMRD profiles reflect the spectrum of the reorientational and the diffusional molecular dynamics. The latter are described by Lorentzian functions with the form reported in equation (6) through which information about correlation functions of the microscopic fluctuations can be achieved ${ }^{6,37}$ :

$$
J\left(\omega_{\mathrm{L}}\right)=\frac{\tau_{\mathrm{C}}}{1+\left(\omega_{\mathrm{L}} \tau_{\mathrm{C}}\right)^{2}}
$$

In equation $(6), J\left(\omega_{\mathrm{L}}\right)$ is the spectral density function describing the distribution of the motion frequencies in a molecular system, $\omega_{\mathrm{L}}$ is the proton Larmor frequency, and $\tau_{\mathrm{C}}$ is the 


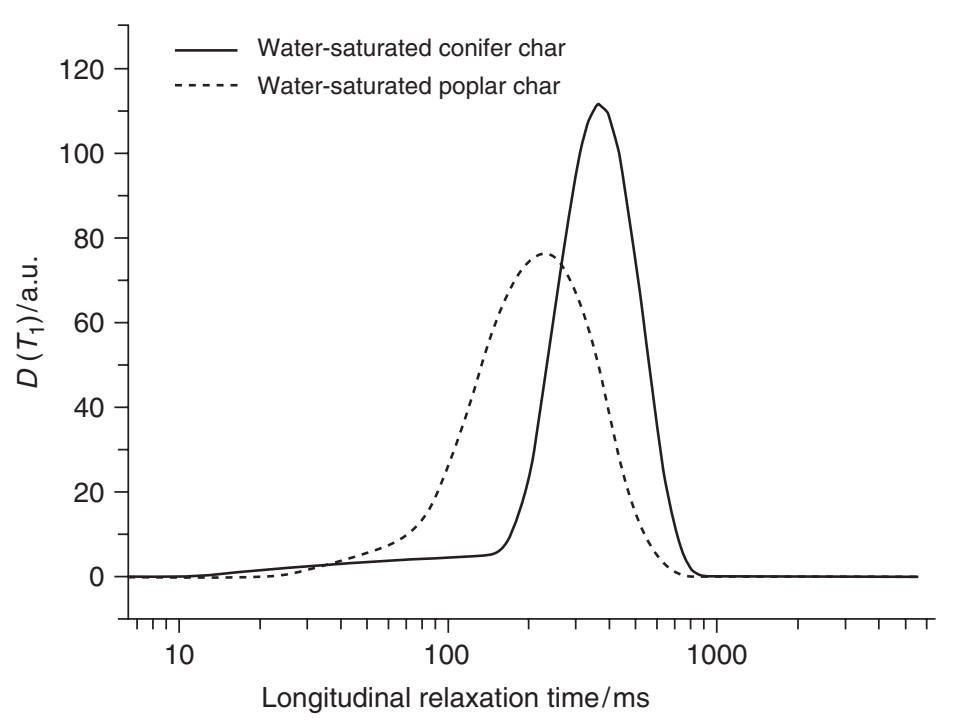

Figure 4. Relaxograms of water-saturated conifer and poplar chars obtained by applying the UPEN algorithm. (Adapted with permission from Ref. 22. (c) Springer-Verlag, 2012)

correlation time. Correlation time describes the random molecular motions of molecular systems either in solution or in porous media. ${ }^{6}$ Namely, $\tau_{C}$ is the time taken for a molecule to rotate one radian or to move a distance of the order of its own dimension. ${ }^{12}$ The longer the $\tau_{\mathrm{C}}$ value is, the slower are the molecular motions, thereby revealing restrictions in the motional freedom degrees of spatially restrained molecular systems. Conversely, as a molecule encompasses faster motions because of higher degrees of freedom in larger spaces, shorter correlation time values are expected.

Equation (6) was redefined by Halle et al., ${ }^{38}$ as in equation (7), to account for the stretching of the NMR dispersion profiles as a consequence of the complexity of the reorientational dynamics within the molecular system, the heterogeneous distributions of proton exchange rates, and the heterogeneous distribution of intermolecular dipole couplings. Equation (7) is also referred to as model-free-analysis ${ }^{38}$ :

$$
J\left(\omega_{\mathrm{L}}\right)=\sum c_{i} \frac{\tau_{\mathrm{C} i}}{1+\left(\omega_{\mathrm{L}} \tau_{\mathrm{C} i}\right)^{2}}
$$

In equation (7), the subscript ' $i$ ' refers to the different components of the motion and $c_{i}$ is a fitting parameter. The summation of $c_{i}$ values represents the mean square fluctuation that contains information about the equilibrium structure of the system, thereby being independent of the system dynamics. ${ }^{38,39}$

The parameters of the multi-Lorentzian equation (7) can be determined by any nonlinear parameter estimation method, while the number $N$ of Lorentzians to be included can be objectively determined by means of any statistical procedure. As an example, the $F$-test has been applied in some papers. ${ }^{22,23,25,38}$ Briefly, a fit of $N$ Lorentzian terms to $M$ data points $J\left(\omega_{i}\right)$ with errors $\sigma_{i}$ provides a $\chi^{2}(N)$ value that must be compared to the $\chi^{2}(N+1)$ value obtained by applying $N+1$ Lorentzians. If the fit improves, the ratio $F_{(N, N+1)}=\chi^{2}(N) / \chi^{2}(N+1)$ increases [i.e., $\left.\chi^{2}(N+1)<\chi^{2}(N)\right]$. The procedure must be iterated until $F_{(N+m-1, N+m)}>F_{(N+m, N+m+1)}$, where $m$ is integer and $\geq 0$. When the latter condition is satisfied, the acceptable number of Lorentzians to be applied in equation (7) is $N+m$.

It must be emphasized that the model proposed by Halle et al. ${ }^{38}$ is only a useful and convenient method to fit the experimental data. The set of parameters $\left\{c_{i}, \tau_{i}\right\}$ obtained by equation (7) does not have any physical significance, unless independent information suggests that the system can actually be modeled by a fixed number of Lorentzians. In the latter case, a direct physical interpretation of the parameters can be attempted. As an example, Prư̌ová et al. ${ }^{40}$ reported the dynamics properties of three water hydration layers (bound, weakly bound, and bulk) surrounding hyaluronan. Presence of these layers around hyaluronan has been previously hypothesized by rheology and thermal analyses. ${ }^{41,42}$

Halle et al. ${ }^{38}$ also showed that each fitting parameter, $c_{i}$, can be considered as a weighing factor for the $i$ th correlation time value, thereby leading to the calculation of a weight-average correlation time $(\langle\tau\rangle)$ according to expression (8):

$$
\langle\tau\rangle=\frac{\sum_{i} c_{i} \tau_{\mathrm{C} i}}{\sum_{i} c_{i}}
$$

The weight-average correlation time retrieved by applying equation (8) describes the average dynamics of the whole complex system under investigation.

Following application of the biexponential form of equations (3), equations (7) and (8) have been used to monitor the dissolution mechanism of crystalline cellulose in phosphoric acid $\left(\mathrm{H}_{3} \mathrm{PO}_{4}\right) \cdot{ }^{14}$ In particular, two forms of $\mathrm{H}_{3} \mathrm{PO}_{4}$ have been recognized. The first one showed the fastest longitudinal relaxation rates in the whole range of $B_{\mathrm{RLX}}$ intensities (i.e., the shortest spin-lattice relaxation times). This $\mathrm{H}_{3} \mathrm{PO}_{4}$ form was (i) considered strongly bound to cellulose because of the formation of phosphor-ester linkages and (ii) responsible for cellulose 
dissolution. Formation of $\mathrm{C}-\mathrm{O}-\mathrm{P}$ bridges allowed cellulose swelling and solvation. The second $\mathrm{H}_{3} \mathrm{PO}_{4}$ form showed the slowest $R_{1}$ values in the NMRD profile, thereby being considered as bulk phosphoric acid. The $\langle\tau\rangle$ values reported by Conte et al. ${ }^{14}$ supported the aforementioned findings. In fact, the correlation time for the bulk phosphoric acid resulted shorter than that retrieved for the strongly bound form of $\mathrm{H}_{3} \mathrm{PO}_{4} \cdot{ }^{14}$

It has been proved that the Bloembergen-Purcell-Pound (BPP) model reported in equation (9) can be successfully applied to fit NMRD profiles in order to reveal the dynamics properties of liquids interacting to the surface of crystalline organic solids. ${ }^{6,43}$ This model applies only to pure compounds when isotropic rotational diffusion of molecules and intramolecular interaction of two-spin- $1 / 2$ system with fixed internuclear distances can be hypothesized. ${ }^{6}$ Equation (7) can be included in equation (9) in order to account for dynamics inhomogeneity in more heterogeneous systems ${ }^{44}$ :

$$
R_{1}=\frac{1}{T_{1}}=\alpha+\beta\left[0.2 J\left(\omega_{\mathrm{L}}\right)+0.8 J\left(2 \omega_{\mathrm{L}}\right)\right]
$$

In equation (9), all the terms have been already defined with the exception of $\alpha$ and $\beta$. The former represents the highfield relaxation rate, whereas the latter is a constant related to the dipolar interactions. Increasing dipolar strengths, due to reduced molecular mobility, produce longer $\alpha$ and larger $\beta$ values. Conversely, weak dipolar couplings are generated by unbound (or freely moving) molecules, thereby providing shorter $\alpha$ and smaller $\beta$ values. ${ }^{38,44}$ As correlation times are a measure of the rate of molecular reorientation in the time unit (see earlier), low $\alpha$ and $\beta$ values due to unconstrained molecular motions are also associated to short $\tau_{\mathrm{C}}$ values. On the other hand, the larger the $\alpha$ and $\beta$ values are, the longer result the correlation times, because of restrictions in molecular mobility.
The model-free BPP analysis was used to differentiate among water-saturated chars obtained from a gasification process applied to marc, poplar, and conifer wastes. ${ }^{22}$ In particular, results revealed that: (i) the water relaxation rates in the NMRD profiles varied in the order $R_{1 \text { (marc char) }}>R_{1 \text { (poplar char) }}>R_{1 \text { (conifer char) }} \quad$ (Figure 5); (ii) the $\alpha$ values were in the order $\alpha_{(\text {marc char })} \approx \alpha_{\text {(poplar char })}>$ $\alpha_{\text {(conifer char); }}$; (iii) the $\beta$ values changed as $\beta_{\text {(marc char) }} \gg$ $\beta_{\text {(conifer char) }} \approx \beta_{\text {(poplar char) }}$; and (iv) the weight-average correlation times varied in the order $\langle\tau\rangle_{\text {(marc char) }}<\langle\tau\rangle_{\text {(conifer char) }}$ $<s\langle\tau\rangle_{\text {(poplar char) }}{ }^{22}$ The fastest $R_{1 \text { (marc char) }}$ and the longest $\beta_{\text {(marc char) }}$ values were due to the presence of paramagnetic impurities. The latter fasten spin-lattice relaxation times and raise up $\beta$ values, thereby altering the results achievable by combining equations (7) and (9). ${ }^{12}$ For this reason, dynamics of water on the surface of marc char could not be compared to that of water moving on poplar and conifer chars. On the other hand, differences between $\alpha, \beta$, and $\langle\tau\rangle$ values for the latter two water-saturated chars were attributed to the different porosities that were, in turn, affected by the nature of the biomass feedstocks. ${ }^{22}$

Porosity was also responsible for the differences among the parameters retrieved by applying the model-free BPP analysis on water-saturated soils ${ }^{24}$ and sediments. ${ }^{25}$ In the first case, NMRD profiles were able to reveal that texture of a gypsic haploxerept soil was affected by the nature of three different plant species used in the afforestation of a Mediterranean area. In the second case, porosity variations associated to different $\alpha$, $\beta$, and $\langle\tau\rangle$ values in the water-saturated layers of a sedimentary core allowed to reconstruct the environmental evolution of a Sicilian (Italy) saltmarsh.

Equations (6)-(9) apply either to liquid mixtures or to liquids moving on the surface of porous media. Conversely, NMRD profiles of solid-state systems are better described by

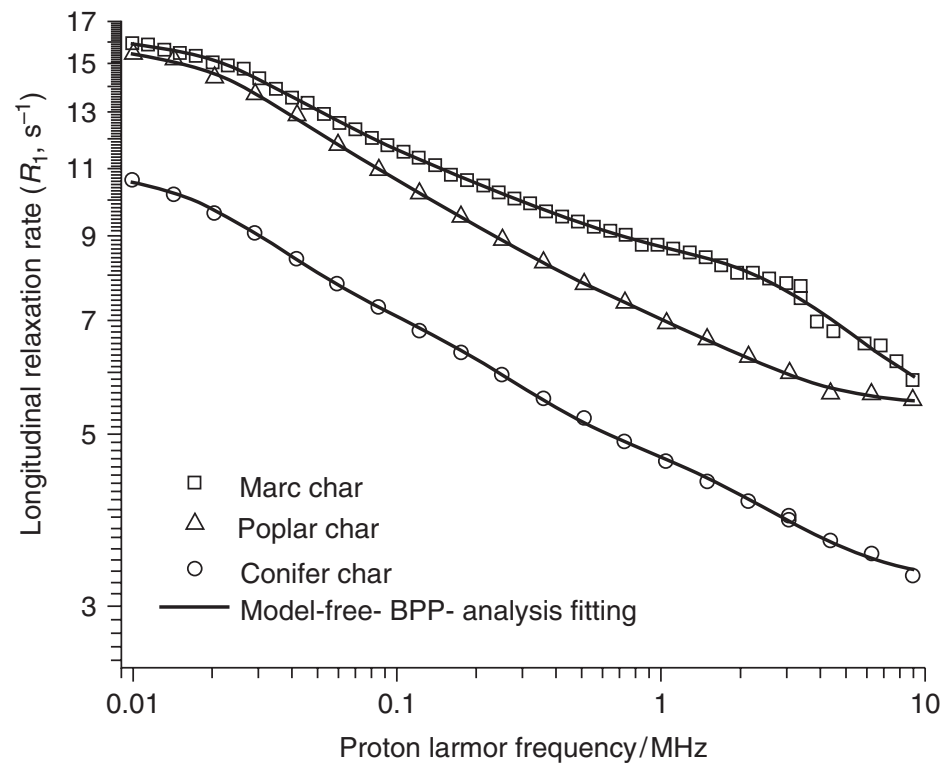

Figure 5. NMRD profiles for water-saturated marc, poplar, and conifer chars fitted by applying the model-free BPP analysis. (Adapted with permission from Ref. 22. ( ) Springer-Verlag, 2012) 
the power-law model reported in equation $(10)^{45}$ :

$$
R_{1}=\frac{1}{T_{1}}=A \omega_{0}^{-b}+C
$$

Here, $A$ is the size of the low-frequency relaxation rate that includes differences in spin density, structural organization, and local internal dynamics, ${ }^{46-48} \omega_{0}$ is the proton Larmor frequency, $b$ is a power factor, and $C$ is the high-field relaxation rate. This model was applied to study the transformation of leaves to litters in a reafforestated soil located in Sicily (Italy). ${ }^{20}$ Results showed that while $A$ changed in the order $A_{\text {leaves }}>A_{\text {litters }}, C$ varied in the order $C_{\text {leaves }}<C_{\text {litters }}$. The trend inversion was attributed to the changes in the motion regimes (see section titled 'The Motion Regimes and the Qualitative Aspects of NMRD Profiles in Environmental Applications and Green Chemistry'), which flip from fast to slow, as the proton Larmor frequency is raised up. ${ }^{12}$ In the slow-motion regime, the faster the rotational motions are (as in litters), the slower is the relaxation efficiency (i.e., $A_{\text {leaves }}>A_{\text {litters }}$ ). Conversely, in the fast-motion regime, the relaxation efficiency increases with the swiftness of the rotational motions (i.e., $C_{\text {leaves }}<C_{\text {litters }}$ ). In addition, as $A$ includes also differences in spin density, the results revealed that spin density in litters is lower than in leaves as a consequence of the degradation mechanisms. The values of the power factor, $b$, changed in the order $b_{\text {leaves }}>b_{\text {litters. }}$. In general, $b$ ranges from 0.45 to 0.75 in solid proteins. ${ }^{6}$ As a matter of fact, hydrated solid proteins reveal $b$ values close to 0.45 ; whereas the lower the hydration degree is, the higher is the power factor value. ${ }^{6,45,49,50}$ In polymer science, it is also reported that changes in $b$ values can be associated to intra- or intersegment relaxation contributions according to the range of values within which $b$ falls. Namely, if $0.20<b<0.33$, intrasegment relaxations prevail, whereas when $0.40<b<0.50$, intersegment relaxations become predominant. ${ }^{6}$ However, Berns et al. ${ }^{20}$ pointed out that, at the moment, a complete theory on the power dependence of NMRD profiles of heterogeneous complex solid systems (such as leaves, litters or, more generally, biomasses and environmental matrices) is still missing. For this reason, the physical meaning of $b$ values from equation (10) applied to environmental systems cannot be provided yet.

\section{The Motion Regimes and the Qualitative Aspects of NMRD Profiles in Environmental Applications and Green Chemistry}

Figure 6 shows the evolution of $T_{1}$ as affected by correlation time and temperature variations. ${ }^{12,51}$ This behavior comes by the Lorentzian shape of the spectral density as reported in equation (6).

The fast-motion regime is typical for liquid systems whose $\tau_{C}$ values lay generally in the interval $10^{-8}$ to $10^{-12} \mathrm{~s}{ }^{51}$ In this condition, $\left(\omega_{0} \tau_{\mathrm{C}}\right)^{2} \ll 1$ and $J\left(\omega_{\mathrm{L}}\right) \propto \tau_{\mathrm{C}}$. For this reason, the longer the $\tau_{\mathrm{C}}$ is, the shorter is the spin-lattice relaxation time. $T_{1}$ reduction follows a monotonic behavior (Figure 6). Then, it turns around a minimum, where $\left(\omega_{0} \tau_{C}\right)^{2} \approx 1$ (intermediatemotion regime) to attain the slow-motion regime, where very large $T_{1}$ values for very large correlation times are retrieved (Figure 6). The condition $\left(\omega_{0} \tau_{C}\right)^{2} \gg 1$, valid for rigid solids, is satisfied in the slow-motion regime. As correlation times are directly related to temperature, switch among the three motion regimes can be also described in terms of $T$ variations (Figure 6).

Variable temperature (VT) FFC NMR relaxometry experiments have been conducted to evaluate the motion regime of water on the surface of two polymorphs of a commercial

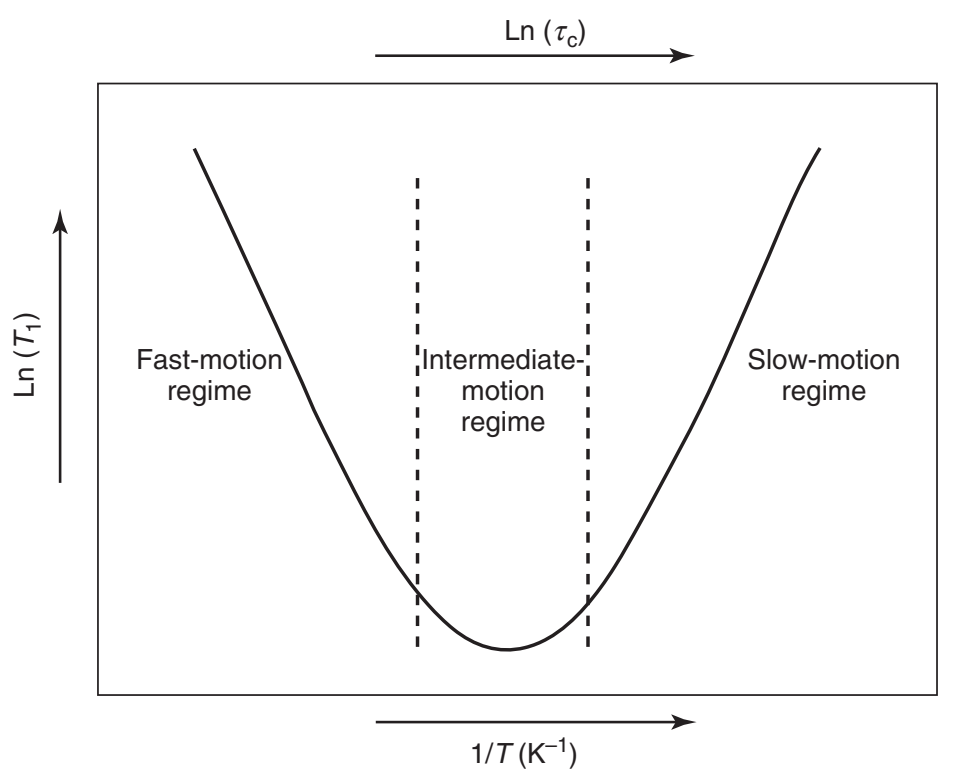

Figure 6. Simulation of the evolution of the longitudinal relaxation time as a function of the temperature $(1 / T)$ and the correlation time $\left(\tau_{\mathrm{C}}\right)$ for a fixed value of the measurement magnetic field frequency 


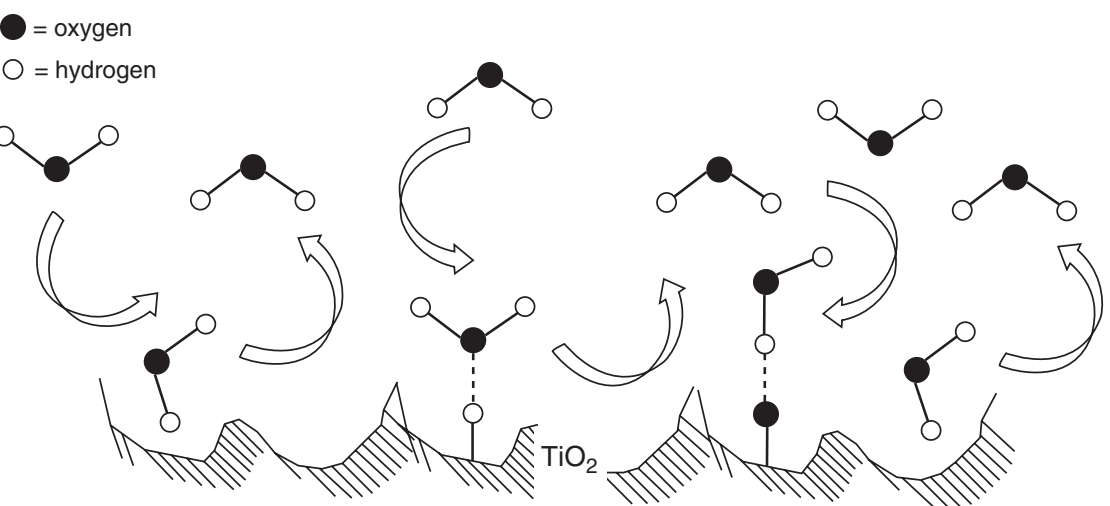

Figure 7. Graphical representation of the water dynamics on the surface of titanium oxide. Water is either retained on the porous surface by hydrogen bonds or not chemically constrained. H-bonds are responsible for the slow-motion regime, whereas freely moving water undergoes fast-motion regime. (Adapted with permission from P. Conte, V. Loddo, C. De Pasquale, V. Marsala, G. Alonzo, and L. Palmisano, J. Phys. Chem. C, 2013, 117, 5269. Copyright 2013 American Chemical Society)

photo-catalyst (rutile and anatase $\mathrm{TiO}_{2}$ ) generally used in green chemistry reactions. ${ }^{26}$ Results revealed that the motion regime changed according to the nature of the two titanium oxides. In particular, water moving on the surface of rutile was subjected to fast-motion regime, whereas that on anatase surface was moving according to the slow-motion regime. The dynamics properties of water on the surface of a nonparamagnetic porous system can be described by equation $(11)^{3,6,52-54}$ :

$$
R_{1}=\frac{1}{T_{1}}=\frac{f_{\mathrm{M}}}{T_{1 \mathrm{M}}+\tau_{\mathrm{M}}}
$$

Here, $f_{\mathrm{M}}$ represents the molar fraction of water chemically bound to porous material, $T_{1 \mathrm{M}}$ is the proton spin-lattice relaxation time of the chemically bound water, and $\tau_{\mathrm{M}}$ is the exchange correlation time, which measures the mean residence time of the bound water. When fast-motion regime prevails (such as on the surface of rutile), the proton spin-lattice relaxation rate is proportional to $1 / T_{1 \mathrm{M}}$, thereby allowing the consideration that water is not chemically retained on the rutile surface. Conversely, as predominance of slow-motion regime is achieved (such as in the case of water moving on anatase surface), the proton spin-lattice relaxation rate depends on $1 / \tau_{M}$ and water lays longer on the anatase surface because of the formation of H-bonds ${ }^{26}$ (Figure 7). The FFC NMR relaxometry findings explained why the two $\mathrm{TiO}_{2}$ polymorphs differ in their photo-reactivity yield. In fact, the mechanism of the photocatalytic oxidation reactions foresees the formation of $\cdot \mathrm{OH}$ radicals that are believed to be responsible for the primary oxidant attack to the substrate. The interaction between adsorbed water and $\mathrm{TiO}_{2}$ surface plays the major role to produce the oxidant species. Consequently, the absence of chemical interactions between $\mathrm{H}_{2} \mathrm{O}$ and the surface of rutile, as highlighted by NMRD results, suggests that the formation of . $\mathrm{OH}$ radicals from $\mathrm{H}_{2} \mathrm{O}$ is highly improbable, thereby making such a catalyst poorly active. ${ }^{26}$

Formation of hydrogen bonds was suggested also to explain the slow-motion regime observed by VT FFC NMR relaxometry experiments for water moving on the surface of a char obtained from an industrial thermochemical process. ${ }^{23}$ In this case, hydrogen bonding involved the electron-deficient water hydrogen atoms and the electron-rich graphite-like systems, thereby leading to the realization of nonconventional $\mathrm{H}$-bond interactions. The presence of weak $\mathrm{H}$-bonds between char and water can explain the large water capacity and structural stability of char amended soils..$^{55}$

Promising information that is relevant for mineral oil industry has also been obtained from VT FFC NMR relaxometry experiments performed to monitor the dynamics of oil in bulk and reservoir rocks. ${ }^{56}$ Results revealed that oil in bulk rocks was subjected to fast-motion regime. Conversely, the presence of hydrophobic sites in the reservoir rocks was hypothesized to explain the more restricted motion of oil, thereby resulting in a slow-motion regime. The hydrophobic oil molecules can be trapped on the surface of reservoir rocks because of Van der Waals interactions.

Temperature and magnetic field variations in relaxometry experiments allowed to reveal solvent-gelator interactions in a mixture made by chlorobenzene and methyl-4,6-O- $(p-$ nitrobenzylidene)- $\alpha$-D-glucopyranoside gel (MPNBG). ${ }^{21}$ In particular, the slow-motion regime observed between 273 and $340 \mathrm{~K}$ was explained by the confinement of chlorobenzene in the MPNBG tri-dimensional network. This confinement was driven by the interactions between the electric dipolar moment of chlorobenzene and the polar MPNBG surface. Importance of such study resides in the possibility to formulate new systems for pesticide synthesis and applications.

\section{Conclusions and Future Perspectives}

While doing literature search for the present article, we realized that the number of FFC NMR relaxometry applications in environmental field is, at the moment, very few and mostly those applications have been cited here. The lack of studies involving FFC NMR relaxometry to evaluate environmental problems and possibly suggest solutions is probably due to the better worldwide distribution of the traditional high-field NMR spectroscopy instruments with emphasis on answering questions that are related to the structure of organic 
matter in soils, water, and atmosphere. ${ }^{57,58}$ Information about molecular dynamics and molecular interactions between contaminants and constituents of environmental compartments can also be achieved in high-field NMR spectroscopy by application of VT experiments. These may induce phase transitions and sample decomposition, thereby altering the experimental results. In addition, the frequency of the molecular motions investigated by high-field NMR spectroscopy downs only to $10^{8} \mathrm{~Hz}$. As evidenced throughout the present article, FFC NMR relaxometry overcomes all the aforementioned problems, although it lacks in resolution and cannot provide structural information concerning the chemical nature of the investigated environmental system. ${ }^{6}$ In addition, model theories for FFC NMR relaxometry have been developed mainly for polymer dynamics such as lipids, proteins, and carbohydrates, ${ }^{5,6,44,59,60}$ material science, ${ }^{53,61-63}$ and contrast agents for magnetic resonance imaging applications. ${ }^{64,65}$ For this reason, FFC NMR relaxometry should be supported by results from other analytical techniques when it is applied in environmental field.

Additional advantages of FFC NMR relaxometry as compared to traditional high-field NMR spectroscopy lie in the nonnecessity of deuterated solvents (locking of magnetic fields is unnecessary); in the cryogen-free technology (the necessity to switch among three different magnetic fields makes cryogen gasses superfluous); in the possibility to monitor molecular dynamics at magnetic field intensities close to that of the Earth's magnetic field (this makes possible investigation of very slow dynamics and, hence, very strong molecular interactions); and in the ability to monitor dynamics of quadrupolar nuclei through indirect detection of NMR sensitive spin- $1 / 2$ nuclei in intact systems. ${ }^{6,8,11}$ The latter point is very important in environmental investigations because of the possibility to monitor fate of chlorinated contaminants by observing the quadrupolar effects of chlorine in polluted soils. In particular, ${ }^{35} \mathrm{Cl}$ quadrupoles are observed in NMRD profiles as quadrupolar dips in the proton Larmor frequency interval $0.3-4.5 \mathrm{MHz}{ }^{61}$ Shape and position of the quadrupolar dips depend on temperature of the system and nature of the interactions between organo-chlorine compounds and their chemical environment. ${ }^{61}$

Quadrupolar effects on relaxometry behavior of hydrogen nuclei in environmental systems can be generated also by ${ }^{14} \mathrm{~N}$ nuclei. ${ }^{6,66}$ The possibility to monitor the environmental behavior of nitrogen-containing molecules through the influence of ${ }^{14} \mathrm{~N}$ on the dynamics of ${ }^{1} \mathrm{H}$ overcomes the classical limit for the high-field NMR spectroscopy. In fact, although relative abundance of ${ }^{14} \mathrm{~N}$ is larger than that of ${ }^{15} \mathrm{~N},{ }^{14} \mathrm{~N}$ NMR spectroscopy is resolutionless as compared to ${ }^{15} \mathrm{~N}$ NMR spectroscopy because of ${ }^{14} \mathrm{~N}$ quadrupole properties. For this reason, high-field NMR spectroscopy on environmental samples is traditionally performed on ${ }^{15} \mathrm{~N}$-enriched materials. ${ }^{67}$ The ${ }^{15} \mathrm{~N}$ enrichment is achieved either by purification ${ }^{67}$ or by growing biomasses with ${ }^{15} \mathrm{~N}$-containing nutrients. ${ }^{68}$ Both procedures are not needed when molecular dynamics investigation is performed by FFC NMR relaxometry. Future advances in FFC NMR relaxometry can be achieved by the hightemperature superconducting technology ${ }^{69}$ In fact, the use of high-temperature superconductors may allow to span a very wide interval of magnetic fields with the possibility to combine together multinuclear relaxometry and spectroscopy in only one instrument.

\section{Acknowledgment}

The authors are very grateful to Dr Salvatore Bubici (Stelar srl) for the fruitful discussions on the relaxometry technique.

\section{Biographical Sketches}

Pellegrino Conte was born in 1967. He graduated in chemistry, 1992 and $\mathrm{PhD}$ in agricultural chemistry, 1998, at the Università degli Studi di Napoli Federico II (Italy). He worked at the NMR Centre of the Italian National Council of Research in 1992. In 1996 and 2000, he guested at the NMR Centre of the Wageningen University (The Netherlands). From 2005 to 2010, he has been periodically a guest scientist at the Forschungszentrum Juelich (Germany). Since 2009, he is responsible for the NMR relaxometry lab at the Università degli Studi di Palermo (Italy). He has contributed approximately 90 papers.

Giuseppe Alonzo was born in 1946. He graduated in chemistry at the Università degli Studi di Palermo (Italy) in 1970. He spent time at Delaware University, USA (1978), University of Leeds, UK (1980), Rutgers University, USA (1983), and Dortmund University, Germany (1984), where he has been working in NQR and Mössbauer spectroscopy. From 2012, he is the chief of Consiglio per la Ricerca e la Sperimentazione in Agricoltura (Rome, Italy). He has contributed approximately 130 publications.

\section{References}

1. A. Abragam, 'Principles of Nuclear Magnetism', 1st edn, Oxford Science Publications, 1962.

2. D. Neuhaus and M. Williamson, 'The Nuclear Overhauser Effect in Structural and Conformational Analysis', VCH Publishers, Inc, 1989.

3. J.-P. Korb, Magn. Reson. Imag., 2001, 19, 363.

4. J. V. Bayer, F. Jaeger, and G. E. Schaumann, Open Magn. Reson. J., 2010, 3, 15.

5. R. Kimmich and N. Fatkullin, Adv. Polymer Sci., 2004, 170, 1.

6. R. Kimmich and E. Anoardo, Progr. Nucl. Magn. Reson. Spectr., 2004, 44, 257

7. R. Kimmich, Bull. Magn. Reson., 1980, 1, 195.

8. E. Anoardo, G. Galli, and G. Ferrante, Applied Magn. Reson., 2001, 20, 365.

9. S. V. Dvinskikh, Instrum. Exp. Tech., 1996, 39, 709.

10. S. Bubici, G. Ferrante, and R. Steele Application note at http://www.stelar.it/2010/download/free/files/AN_120901-NMRDHeteroNUc.pdf

11. G. Ferrante and S. Sykora, Adv. Inorg. Chem., 2005, 57, 405.

12. V. I. Bakhmutov, 'Practical NMR Relaxation for Chemists', Wiley, 2004.

13. D. Canet, Progr. Nucl. Magn. Reson. Spectr., 1989, 21, 237.

14. P. Conte, A. Maccotta, C. De Pasquale, S. Bubici, and G. Alonzo, J. Agric. Food Sci., 2009, 57, 8748

15. F. Vaca Chàvez and B. Halle, Magn. Reson. Med., 2006, 56, 73.

16. J. R. Melton, A. Kantzas, and C. H. Langford, Anal. Chim. Acta, 2007, 605, 46.

17. C. L. Bray, R. G. Bryant, M. J. Cox, G. Ferrante, Y. A. Goddard, S. K. Sur, and J. P. Hornak, J. Environ. Eng. Geoph., 2009, 14, 49. 
18. P. Conte, S. Bubici, E. Palazzolo, and G. Alonzo, Spectr. Lett., 2009, 42, 235.

19. A. Pohlmeier, S. Haber-Pohlmeier, and S. Stapf, Vadose Zone J., 2009, 8 735.

20. A. E. Berns, S. Bubici, C. De Pasquale, G. Alonzo, and P. Conte, Org. Geochem., 2011, 42, 978

21. J. Tritt-Goc, M. Bielejewski, and R. Luboradzki, Tetrahedron, 2011, 67, 8170.

22. C. De Pasquale, V. Marsala, A. E. Berns, M. Valagussa, A. Pozzi, G. Alonzo, and P. Conte, J. Soils Sediments, 2012, 12, 1211.

23. P. Conte, V. Marsala, C. De Pasquale, S. Bubici, M. Valagussa, A. Pozzi, and G. Alonzo, GCB Bioenergy, 2013, 5, 116.

24. V. A. Laudicina, C. De Pasquale, P. Conte, L. Badalucco, G. Alonzo, and E. Palazzolo, J. Soils Sediments, 2012, 12, 1222

25. A. Maccotta, C. De Pasquale, A. Caruso, C. Cosentino, G. Alonzo, and P. Conte, Environ. Sci. Poll. Res., 2013. On-line-first. DOl: 10.1007/s11356012-1445-4

26. P. Conte, V. Loddo, C. De Pasquale, V. Marsala, G. Alonzo, and L. Palmisano, J. Phys. Chem. C, 2013, 117, 5269

27. L. A. Morozova-Roche, J. A. Jones, W. Noppe, and C. M. Dobson, J. Mol. Biol., 1999, 289, 1055.

28. P. Conte, C. Abbate, A. Baglieri, M. Nègre, C. De Pasquale, G. Alonzo, and M. Gennari, Org. Geoch., 2011, 42, 972

29. W. Provencher, Computer Phys. Commun., 1982, 27, 213.

30. W. Provencher, Comp. Phys. Commun., 1982, 27, 229.

31. G. C. Borgia, R. S. J. Brown, and P. Fantazzini, J. Magn. Reson., 1998, 132, 65.

32. G. C. Borgia, R. S. J. Brown, and P. Fantazzini, J. Magn. Reson., 2000, 147, 273.

33. G. C. Borgia, R. S. J. Brown, and P. Fantazzini, Magn. Reson. Imag., 2001, 19, 473 .

34. S. Haber-Pohlmeier, S. Stapf, D. van Dusschoten, and A. Pohlmeier, Open Magn. Reson. J., 2010, 3, 57.

35. G. E. Schaumann, D. Diehl, M. Bertmer, A. Jaeger, P. Conte, G. Alonzo, and J. Bachmann, J. Hydrol. Hydromech., 2013, 1, 50

36. G. Butera, C. De Pasquale, A. Maccotta, G. Alonzo, and P. Conte, Cellulose, 2011, 18, 1499

37. A. M. Albano, P. A. Beckmann, M. E. Carrington, F. A. Fusco, A. E. O'Neil, and M. E. Scott, J. Phys. C: Solid State Phys., 1983, 16, L979.

38. B. Halle, H. Johannesson, and K. Venu, J. Magn. Reson., 1998, 135, 1.

39. M. Dobies, M. Kozak, and S. Jurga Solid, State Nucl. Magn. Reson., 2004, 25, 188

40. A. Prưšová, P. Conte, J. Kučerik, and G. Alonzo, Anal. Bioanal. Chem., 2010, 397, 3023

41. E. Fouissac, M. Milas, and M. Rinaudo, Macromolecules, 1993, 26, 6945.
42. M. K. Cowman and S. Matsuoka, Carbohydr. Res., 2005, 340, 791.

43. Y. L. Wang and P. S. Belton, Chem. Phys. Let., 2000, 325, 33.

44. C. Luchinat and G. Parigi, Applied Magn. Reson., 2008, 34, 379.

45. W. Nusser, R. Kimmich, and F. Winter, J. Phys. Chem., 1988, 92, 6808.

46. E. E. Burnell, D. Capitani, C. Casieri, and A. L. Segre, J. Phys. Chem. B, 2000, 104, 8782 .

47. E. Murray, D. Carty, P. C. Innis, G. G. Wallace, and D. F. Brougham, J. Phys Chem., 2008, 112, 17688

48. N. Fatkullin, A. Gubaidullin, and S. Stapf, J. Chem. Phys., 2010, 132, 094903.

49. R. G. Bryant, D. A. Mendelson, and C. C. Lester, Magn. Reson. Med., 1991, 21, 117.

50. J.-P. Korb and R. G. Bryant, Magn. Reson. Med., 2002, 48, 21.

51. D. Canet, Adv. Inorg. Chem., 2005, 57, 3.

52. R. B. Lauffer, Chem. Rev., 1987, 87, 901.

53. J.-P. Korb and R. G. Bryant, Adv. Inorg. Chem., 2005, 57, 293.

54. S. Laurent, D. Forge, M. Port, A. Roch, C. Robic, L. V. Elst, and R. N. Muller, Chem. Rev., 2008, 108, 2064

55. B. Glaser, J. Lehmann, and W. Zech, Biol. Fertil. Soils, 2002, 35, 219.

56. J.-P. Korb, S. Godefroy, and M. Fleury, Magn. Reson. Imag., 2003, 21, 193.

57. A. J. Simpson, D. J. McNally, and M. J. Simpson, Progr. Nucl. Magn. Reson. Spectrosc., 2011, 58, 97.

58. A. J. Simpson, M. J. Simpson, and R. Soong, Environ. Sci. Technol., 2012, 46, 11488 .

59. B. P. Hills, Y. L. Wang, and H.-R. Tang, Mol. Phys., 2001, 99, 1679.

60. G. Kassab, D. Petit, J.-P. Korb, T. Tajouri, and P. Levitz, Comptes Rendus Chimie, 2006, 9, 493.

61. D. J. Pusiol and E. Anoardo, Braz. J. Phys., 1998, 28, 1.

62. J.-P. Korb, L. Monteilhet, P. J. McDonald, and J. Mitchell Cement, Concrete Res., 2007, 37, 295.

63. S. Muncaci, C. Mattea, S. Stapf, and I. Ardelean, Magn. Reson. Chem., 2013, 51, 123.

64. T. Vitha, V. Kubiček, J. Kotek, P. Hermann, L. Vander Elst, R. N. Muller, I. Lukeš, and J. A. Peters, Dalton Trans., 2009, 17, 3204.

65. D. Ó. Hógáin, G. R. Davies, S. Baroni, S. Aime, and D. J. Lurie, Phys. Med. Biol., 2011, 56, 105.

66. J. Seliger and V. Žagar, J. Magn. Reson., 2008, 193, 54.

67. M. W. I. Schmidt, H. Knicker, P. G. Hatcher, and I. Kögel-Knaber, Eur. J. Soil Sci., 1997, 48, 319

68. R. J. Smernik and J. A. Baldock, Plant Soil, 2005, 275, 271.

69. Y. Yeshurun, A. P. Malozemoff, and A. Shaulov, Rev. Modern Phys., 1996 68, 911. 